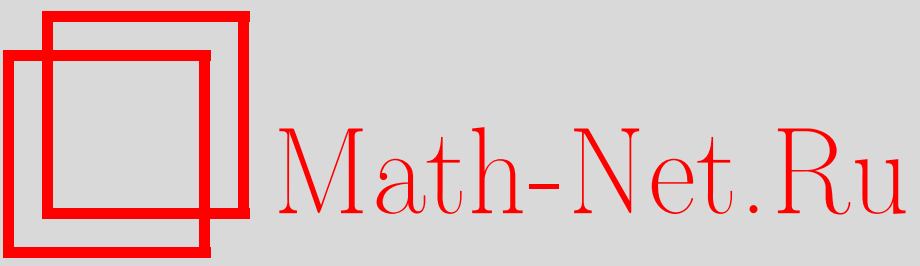

М. Н. Афанасьева, Е. Б. Кузнецов, Метод непрерывного продолжения по параметру при решении краевых задач для нелинейных систем дифференциальноалгебраических уравнений с запаздыванием, имеющих особые точки, Итоги науки и техн. Сер. Соврем. мат. и ее прил. Темат. обз., 2021, том 192, 38-45

DOI: https://doi.org/10.36535/0233-6723-2021-192-38-45

Использование Общероссийского математического портала Math-Net.Ru подразумевает, что вы прочитали и согласны с пользовательским соглашением

http: //www. mathnet.ru/rus/agreement

Параметры загрузки:

IP : 3.85 .183 .62

26 апреля 2023 г., 12:20:56 


\title{
МЕТОД НЕПРЕРЫВНОГО ПРОДОЛЖЕНИЯ ПО ПАРАМЕТРУ ПРИ РЕШЕНИИ КРАЕВЫХ ЗАДАЧ ДЛЯ НЕЛИНЕЙНЫХ СИСТЕМ ДИФФЕРЕНЦИАЛЬНО-АЛГЕБРАИЧЕСКИХ УРАВНЕНИЙ С ЗАПАЗДЫВАНИЕМ, ИМЕЮЩИХ ОСОБЫЕ ТОЧКИ
}

\author{
(c) 2021 г. \\ М. Н. АФАНАСЬЕВА, Е. Б. КУЗНЕЦОВ
}

\begin{abstract}
АннотАция. Рассматривается численный метод решения нелинейной краевой задачи для системы дифференциально-алгебраических уравнений с запаздывающим аргументом, имеющих предельные особые точки. Для численного решения краевой задачи применяется метод стрельбы. Значение параметра «пристрелки» вычисляется с помощью метода Ньютона. Рассматривается случай, когда задача является плохо обусловленной, вследствие чего метод может расходиться. В этом случае решение строится продвижением по наилучшему параметру, которым является длина кривой множества решений. Решение начальной задачи при каждом найденном значении параметра «пристрелки» вычисляется с помощью метода непрерывного продолжения по наилучшему параметру.
\end{abstract}

Ключевые слова: численный метод, краевая задача, дифференциальное уравнение с запаздыванием, метод стрельбы, метод продолжения по наилучшему параметру, сингулярно возмущенное уравнение.

\section{THE METHOD OF CONTINUOUS CONTINUATION BY A PARAMETER FOR SOLVING BOUNDARY-VALUE PROBLEMS FOR NONLINEAR SYSTEMS OF DIFFERENTIAL-ALGEBRAIC EQUATIONS WITH DELAY THAT HAVE SINGULAR POINTS}

\author{
(c) 2021 M. N. AFANASIEVA, E. B. KUZNETSOV
}

\begin{abstract}
In this paper, we consider a numerical method for solving a nonlinear boundary-value problem for a system of differential-algebraic equations with a delayed argument that have singular limit points. For a numerical solution of the boundary-value problem, the shooting method is used. The value of the shooting parameter is calculated by the Newton method. We consider the case where the problem is ill-posed and hence the method may diverge. In this case, the solution is constructed by the method of the best parameter, namely, the length of the curve of the set of solutions. The solution of the initial problem for each value of the shooting parameter is calculated using the method of continuous continuation by the best parameter.
\end{abstract}

Keywords and phrases: numerical method, boundary-value problem, differential equation with delay, shooting method, method of continuation by the best parameter, singularly perturbed equation.

AMS Subject Classification: 34B16

Работа выполнена при поддержке Российского научного фонда (проект № 18-19-00474). 
1. Введение. Множество явлений в физике и механике моделируются нелинейными краевыми задачами для дифференциальных уравнений с запаздыванием. Системы подобного типа описывают процессы механики, зависящие не только от характеристик рассматриваемых параметров в текущий момент времени, но также и от исследуемых характеристик в предшествующий момент времени. Результатом исследования, представленного в работе, является алгоритм численного решения подобных задач, сочетающий в себе комбинацию численных методов для решения краевых задач [1] и метода продолжения по наилучшему параметру [9], позволяющих наиболее точно найти возможные решения поставленной задачи.

Методы решения линейных краевых задач для дифференциальных уравнений с отклоняющимся аргументом приведены в [5]. Применение наилучшей параметризации при решении краевых задач для нелинейных обыкновенных дифференциальных уравнений приведено в [6]. Решение краевых задач для систем интегродифференциально-алгебраических уравнений без запаздывания с применением наилучшей параметризации рассмотрено в [2]. В работе [3] описывается применение наилучшей параметризации при решении краевой задачи для нелинейных дифференциально-алгебраических уравнений без запаздывания, приведены примеры работы алгоритма для сингулярно возмущенной краевой задачи. В [4] рассмотрено решение начальных задач для систем интегродифференциально-алгебраических уравнений с запаздыванием с помощью дискретного и непрерывного продолжения по наилучшему параметру.

2. Постановка задачи. Рассматривается система уравнений:

$$
\begin{aligned}
& \epsilon \frac{d y}{d t}=f_{1}(t, y(t), y(t-\tau), \dot{y}(t-\tau), x(t), x(t-\tau), \dot{x}(t-\tau), z(t), z(t-\tau), \dot{z}(t-\tau))=0, \\
& G(t, y(t), y(t-\tau), x(t), x(t-\tau), z(t), z(t-\tau))=0, \quad t \in[a, b], \\
& \frac{d z_{i}}{d t}=f_{2 i}(t, y(t), y(t-\tau), \dot{y}(t-\tau), x(t), x(t-\tau), \dot{x}(t-\tau), z(t), z(t-\tau), \dot{z}(t-\tau))=0,
\end{aligned}
$$

с заданными краевыми условиями:

$$
W(y(a), y(b), x(a), x(b), z(a), z(b))=0,
$$

где

$$
\begin{gathered}
\epsilon \ll 1, \quad i=\overline{1, q}, \quad y(t): \mathbb{R}^{1} \rightarrow \mathbb{R}^{s}, \quad x(t): \mathbb{R}^{1} \rightarrow \mathbb{R}^{r}, \quad z(t): \mathbb{R}^{1} \rightarrow \mathbb{R}^{q}, \\
f_{1}: \mathbb{R}^{1+3 s+3 r+3 q} \rightarrow \mathbb{R}^{s}, \quad f_{2}: \mathbb{R}^{1+3 s+3 r+3 q} \rightarrow \mathbb{R}^{q}, \quad G: \mathbb{R}^{1+2 s+2 r+2 q} \rightarrow \mathbb{R}^{r}, \quad W: \mathbb{R}^{2 s+2 r+2 q} \rightarrow \mathbb{R}^{s+q} .
\end{gathered}
$$

На множестве $E_{0}=\{T<a \mid \exists t>a, t-\tau=T\}$ заданы достаточно гладкие вектор-функции $\varphi_{1,2}(t), \psi_{1,2}(t), \theta_{1,2}(t)$, удовлетворяющие следующим условиям:

$$
\begin{array}{ll}
y_{\tau}=y(t-\tau)=\varphi_{1}(T), & \dot{y}_{\tau}=\dot{y}(t-\tau)=\varphi_{2}(T), \\
x_{\tau}=x(t-\tau)=\psi_{1}(T), & \dot{x}_{\tau}=\dot{x}(t-\tau)=\psi_{2}(T), \\
z_{\tau}=z(t-\tau)=\theta_{1}(T), & \dot{z}_{\tau}=\dot{z}(t-\tau)=\theta_{2}(T) .
\end{array}
$$

Целью работы является построение алгоритма, позволяющего найти и построить возможные решения задачи (1)-(2).

\section{3. Численное решение.}

3.1. Решение краевой задачи. Метод стрелъбы. Согласно принципу метода пристрелки краевая задача (1)-(2) преобразуется к начальной заменой краевого условия в конечной точке $b$ интервала интегрирования на начальное условие путем ввода параметра $p$ :

$$
\begin{array}{lll}
y_{l}(a)=p_{l}, & l=\overline{1, s_{1}}, & s_{1} \leqslant s, \\
z_{m}(a)=p_{s+m}, & m=\overline{1, q_{1}}, & q_{1} \leqslant q .
\end{array}
$$

Теперь искомое решение полученной начальной задачи зависит от параметра $p$ :

$$
y=y(t, p), \quad x=x(t, p), \quad z=z(t, p) .
$$


При этом для функций (5) должно выполняться условие (2), которое с учетом преобразований примет вид

$$
\check{F}(p)=W(y(a, p), y(b, p), x(a, p), x(b, p), z(a, p), z(b, p))=0 .
$$

Значение параметра $p$ должно быть подобрано таким образом, чтобы в конечной точке интервала интегрирования выполнялось граничное условие.

Так как при каждом найденном значении $p$ решается начальная задача, целесообразно использовать методы, обладающие наибольшей скоростью сходимости. Для вычисления значения параметра $p$ применяется метод Ньютона:

$$
\begin{gathered}
p^{(k+1)}=p^{(k)}-\left[\frac{\check{F}\left(p^{(k)}\right)-\check{F}\left(p^{(k-1)}\right)}{p^{(k)-p^{(k-1)}}}\right]^{-1} \check{F}\left(p^{(k)}\right), \\
p^{(0)}=p_{0} .
\end{gathered}
$$

Для успешной сходимости метода Ньютона (7) необходимо выбрать начальное приближение $p_{0}$ достаточно близко к корню, что не всегда представляется возможным. Для преодоления подобных трудностей в [8] предлагается преобразовать систему путем ввода нового параметра $\mu \in[0,1]$ такого, чтобы при $\mu=0$ решение системы было известно или легко определялось, а при $\mu=1$ получалось исходное уравнение с искомым решением:

$$
\Phi(p, \mu)=\check{F}(p)-(1-\mu) \check{F}\left(p_{0}\right)=0,
$$

$p_{0}$ - решение при $\mu=0$. Система (8) решается дискретным методом продолжения по параметру в форме Лаэя (см. [10]). Отрезок, на котором изменяется параметр $\mu$ разбивается на $m$ равных частей:

$$
0=\mu_{1}<\ldots<\mu_{m}=1
$$

Для каждого $\mu_{k}$ вычисляется $p_{k}$ с помощью метода Ньютона:

$$
\begin{gathered}
p_{(k)}^{(i+1)}=p_{(k)}^{(i)}-\left[\frac{\Phi\left(p_{(k)}^{(i)}, \mu_{(k)}\right)-\Phi\left(p_{(k)}^{(i-1)}, \mu_{(k)}\right)}{p_{(k)}^{(i)}-p_{(k)}^{(i-1)}}\right]^{-1} \Phi\left(p_{(k)}^{(i)}, \mu_{(k)}\right) \\
p_{(k+1)}^{(0)}=p_{(k)}^{\left(r_{k}\right)}, \quad i=1,2, \ldots, r_{k-1} .
\end{gathered}
$$

Использование данного подхода позволяет найти решения задачи (1)-(2), однако, оно может быть получено только в том случае, когда решение монотонно зависит от параметра $\mu$.

Для обеспечения нахождения возможных решений задачи (1)-(2), алгоритм преобразуется путем ввода нового параметра $\nu$ - длины кривой множества решений (см. [6]).

Кривая множества решений разбивается на $l$ участков с постоянным шагом:

$$
\nu_{0}=0<\nu_{1}<\nu_{1}<\ldots<\nu_{l}=L .
$$

Теперь неизвестные, входящие в систему (9), являются функциями параметра $\nu$, становятся равноправными и могут меняться немонотонно. Значения $p$ и $\mu$ в $(k+1)$-й точке находятся из решения системы

$$
\Psi_{k+1}(\check{z})=\left\{\begin{array}{l}
\check{F}(p)-(1-\mu) \check{F}\left(p_{0}\right)=0, \\
\left(p-p_{k}^{\left(r_{k}\right)}\right)^{2}+\left(\mu-\mu_{k}^{\left(r_{k}\right)}\right)^{2}-\Delta_{\nu}^{2}=0,
\end{array}\right.
$$

где $\check{z}=(p, \mu), \nu_{k+1}=\nu_{k}+\Delta_{\nu}, \nu_{0}=0, \nu_{l}=L$.

При каждом новом значении параметра $\nu$ система (10) решается методом Ньютона:

$$
\begin{gathered}
\check{z}_{k}^{(i+1)}=\check{z}_{k}^{(i)}-\left[\frac{\partial \Psi\left(\check{z}_{k}^{(i)}\right)}{\partial \check{z}}\right]^{-1} \Psi_{k}\left(\check{z}_{k}^{(i)}\right), \quad i=1,2, \ldots, r_{k-1}, \\
\check{z}_{k}^{(0)}=2 \check{z}_{k-1}^{\left(r_{k-1}\right)}-\check{z}_{k-2}^{\left(r_{k-2}\right)} .
\end{gathered}
$$

При очередном найденном значении параметра $p$ решается задача Коши, от решения которой зависит как вычисление значений параметра $p$, так и непосредственно отыскание решений задачи. Таким образом, выбранный метод решения начальной задачи должен позволять вычислить 
решение даже в случае наличия особых точек. Для решения начальной задачи рассматривается метод непрерывного продолжения по наилучшему параметру.

3.2. Решение начальной задачи. Метод непрерывного продолжения по наилучшему параметру. Начальная задача (1), (4) преобразуется к наилучшему аргументу $\lambda: y=y(\lambda), x=x(\lambda), z=z(\lambda)$, $t=t(\lambda)$. Полагая, что кривая, задаваемая данными функциями, является гладкой, вводятся соотношения:

$$
\begin{aligned}
& \frac{d y}{d \lambda}=Y, \quad \frac{d x}{d \lambda}=X, \quad \frac{d z}{d \lambda}=Z, \quad \frac{d t}{d \lambda}=T, \\
& Y=\left(Y_{1}, \ldots, Y_{s}\right)^{T}, \quad X=\left(X_{1}, \ldots, X_{r}\right)^{T}, \quad Z=\left(Z_{1}, \ldots, Z_{k}\right)^{T} .
\end{aligned}
$$

После дифференцирования по $\lambda$ вектор-функции $G$ и учитывая (12), система (1) примет вид:

$$
\begin{gathered}
Y_{i}-f_{1} T=0, \quad i=\overline{1, s} \\
\sum_{i=1}^{s} G_{y_{i}} Y_{i}+\sum_{j=1}^{r} G_{x_{j}} X_{j}+\sum_{k=1}^{q} G_{z_{k}} Z_{k}+\left(\sum_{i=1}^{s} G_{y_{i \tau}} \dot{y}_{i \tau}+\sum_{j=1}^{r} G_{x_{j \tau}} \dot{x_{j \tau}}+\sum_{l=1}^{q} G_{z_{k \tau}} \dot{z}_{l \tau}+G_{t}\right) T=0, \\
Z_{l}-f_{2 l} T=0, \quad l=\overline{1, q}, \\
\sum_{i=1}^{s} Y_{i} Y_{i}+\sum_{j=1}^{r} X_{j} X_{j}+\sum_{k=1}^{q} Z_{k} Z_{k}+T T=1 .
\end{gathered}
$$

Начальные условия преобразуются к виду

$$
\begin{array}{cl}
y(0)=y_{0}, \quad x(0)=x_{0}, \quad z(0)=z_{0}, \quad t(0)=t_{0}, \\
y(\lambda)=\tilde{y}(\lambda), \quad \dot{y}=\hat{y}(\lambda), \quad \lambda \in\left[-\lambda_{\tau}, 0\right), \\
x(\lambda)=\tilde{x}(\lambda), \quad \dot{x}=\hat{x}(\lambda), \quad \lambda \in\left[-\lambda_{\tau}, 0\right), \\
z(\lambda)=\tilde{z}(\lambda), \quad \dot{z}=\hat{z}(\lambda), \quad \lambda \in\left[-\lambda_{\tau}, 0\right),
\end{array}
$$

где $\lambda_{\tau}$ определяется из решения уравнения $t_{0}-\tau=t\left(-\lambda_{\tau}\right), y_{0}, x_{0}, z_{0}-$ значения соответствующих функций в начальной точке интервала интегрирования $t_{0}=a$, вычисленные в ходе очередной итерации метода стрельбы (4)-(11).

Уравнения (13) задают в пространстве $\mathbb{R}^{1+s+r+q}$ вектор, касательный к интегральной кривой. Согласно уравнению (14) этот вектор является единичным.

Система (13)-(14) ввиду последнего уравнения является нелинейной, что осложняет вычисление значений $Y, X, Z, T$. При нахождении решения в $n$-й точке интегральной кривой можно использовать единичный вектор, касательный к интегральной кривой в предыдущей $n-1$-й точке. При достаточно малом шаге интегрирования $\Delta \lambda_{n}=\lambda_{n+1}-\lambda_{n}$ этот вектор будет близок к вектору, обеспечивающему наилучший аргумент задачи. Поэтому линеаризуем четвертое уравнение системы, приняв его в виде:

$$
\sum_{i=1}^{s} Y_{i}^{*} Y_{i}+\sum_{j=1}^{r} X_{j}^{*} X_{j}+\sum_{k=1}^{q} Z_{k}^{*} Z_{k}+T^{*} T=1,
$$

где $U^{*}=\left(Y^{*}, X^{*}, Z^{*}, T^{*}\right)$ - единичный вектор решения размерности $(s+r+q+1)$, вычисленный на предыдущем шаге.

Вектор $U=(Y, X, Z, T)$, удовлетворяющий системе линейных уравнений (13), (16) не будет единичным, как это требует уравнение (14). Поэтому следует произвести нормировку найденного решения системы (13), (16) по формулам

$$
\tilde{U}_{i}=U_{i} / \sqrt{\sum_{j=1}^{s+r+q+1} U_{j}^{2}}, \quad i=\overline{1, s+r+q+1} .
$$

Таким образом, после преобразования системы (1)-(4) к наилучшему аргументу $\lambda$, который отсчитывается от начальной точки задачи (1), осуществляется переход к системе (12), которая интегрируется при начальных условиях (??). Для решения данной системы применяется метод 
Эйлера с постоянным шагом $h$. При наличии предельных точек используется шаг $h^{*}=t^{*}-t^{(n)}$, где $t^{*}-$ предельная точка, находящаяся в интервале $\left(t^{(n)}, t^{(n+1)}\right)$, где $t^{(n+1)}=t^{(n)}+h$.

Правые части системы (12) вычисляются из решения системы линейных уравнений (13), (16) методом Гаусса и последующей нормировки по формулам (17).

Особенностью рассматриваемой задачи является наличие параметра запаздывания $\tau$. При $t_{\tau}=$ $(t(\lambda)-\tau)$ принадлежащем интервалу, определенному начальными условиями задачи (3), значения функций определены условиями, и дополнительных вычислений производить не требуется. В противном случае для отыскания значения функции в точке запаздывания строится интерполяционный полином Лагранжа по трем точкам. В случае, когда точка $t_{\tau}=(t(\lambda)-\tau)$ совпадает с узловой точкой, вычисленной на предыдущих шагах решения начальной задачи, используется значение функции, вычисленное в этой точке на предыдущих шагах процедуры интегрирования.

\section{4. Численные исследования.}

Пример 1. Рассматривается краевая задача

$$
\left\{\begin{array}{l}
\frac{d y}{d t}=y(t)-2 z(t)-\frac{x(t)}{y^{2}(t-\tau)} \\
\frac{d z}{d t}=z(t-\tau)-2 y(t)+x(t) \\
x(t)-y(t)+t^{2}-4=0 \\
y(t)=\sqrt[3]{2 t+1}, \quad t \in[-\tau, 0) \\
z(t)=0, \quad t \in[-\tau, 0] \\
y(0,6)=-4 \\
\tau=1
\end{array}\right.
$$

После перехода к начальной задаче согласно методу стрельбы и преобразования к наилучшему аргументу $\lambda$, система примет вид:

$$
\left\{\begin{array}{l}
Y-\left(y(t)-2 z(t)-\frac{x(t)}{y^{2}(t-\tau)}\right) \cdot T=0 \\
Z_{l}-(z(t-\tau)-2 y(t)+x(t)) \cdot T=0 \\
-y \cdot Y+x \cdot X+2 t \cdot T=0 \\
Y^{*} \cdot Y+X^{*} \cdot X+Z^{*} \cdot Z+T^{*} \cdot T=1 \\
y(t)=\sqrt[3]{2 t+1}, \quad t \in[-1,0) \\
z(t)=0, \quad t \in[-1,0] \\
t(0)=0 \\
y(0)=p
\end{array}\right.
$$

Графики решения $y(t), z(t)$ и $x(t)$ при значении параметра $p=1,9928$, найденного методом непрерывного продолжения по наилучшему параметру с шагом метода Эйлера $h=0,0001$, представлены на рис. 1-3. На графиках функций $y(t)$ и $x(t)$, в окрестности точки $t=0,5$ касательная ортогональна оси $t$, поэтому обычные численные методы при прохождении данной точки могут расходиться. Использование метода непрерывного продолжения по наилучшему параметру при решении начальной задачи позволило вычислить решение. 


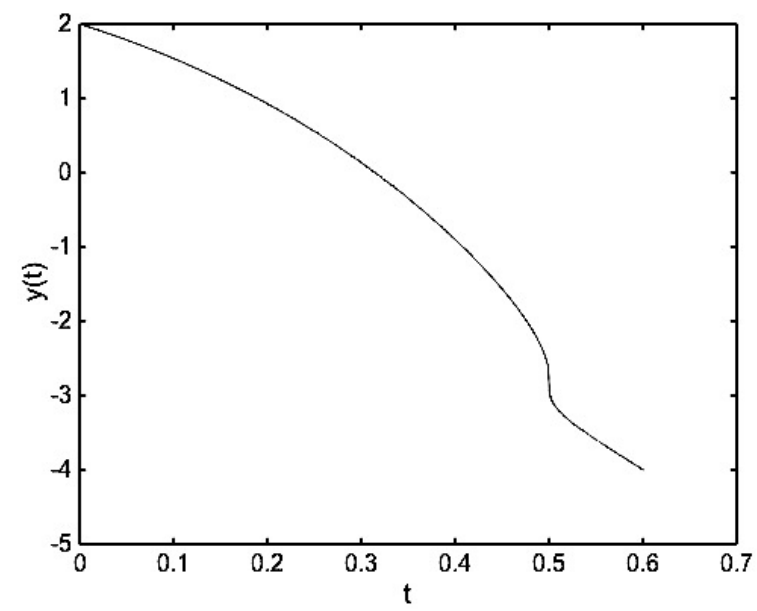

Рис. 1. График решения $y(t)$

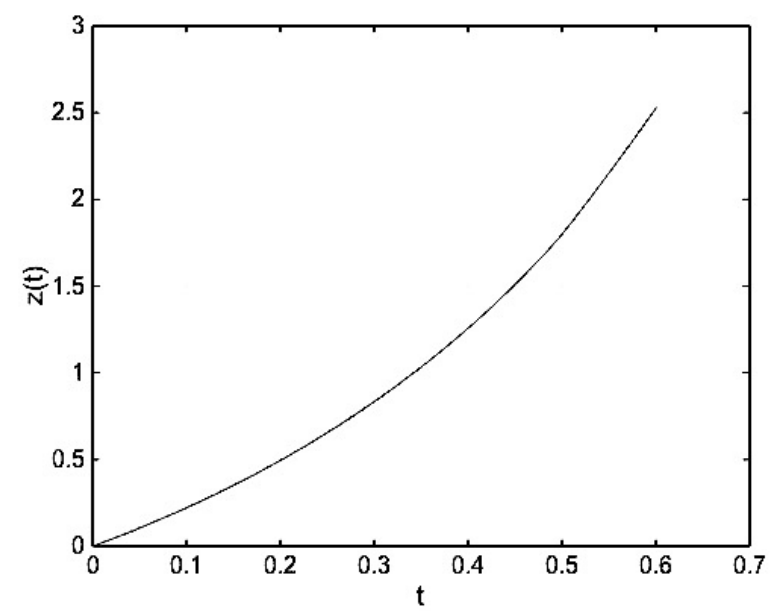

Рис. 2. График решения $z(t)$

Пример 2. Рассматривается сингулярно возмущенная краевая задача

$$
\left\{\begin{array}{l}
\epsilon \cdot y^{\prime \prime}(t)=y(t-\tau)-x^{2}(t) \\
x(t)-y^{2}(t)=0 \\
y(t)=1, \quad t \in[0-\tau, 0] \\
y(1)=0,8, \\
\epsilon=0,05, \quad \tau=0,05
\end{array}\right.
$$

Преобразованная к наилучшему аргументу $\lambda$ задача с введенным параметром «пристрелки» $p$ имеет вид

$$
\left\{\begin{array}{l}
Y-z \cdot T=0 \\
Z-\frac{1}{\epsilon} \cdot\left(y_{\tau}-x^{2}\right) \cdot T=0 \\
x \cdot X-2 y \cdot Y=0, \\
Y^{*} \cdot Y+X^{*} \cdot X+Z^{*} \cdot Z+T^{*} \cdot T=1, \\
y(t)=1, \quad t \in[0-\tau, 0] \\
z(0)=p .
\end{array}\right.
$$

На рис. 4 представлен график функции $y(1, p)$ - значения $y(t)$ в конечной точке интервала интегрирования $b=1$ при значениях параметра $p$ на каждом шаге метода стрельбы при начальном значении $p_{0}=-4$ с применением метода продолжения по параметру для вычисления параметра $p$. График иллюстрирует, что условие $y(1, p)=0,8$ выполняется при $p=-3,8655$ и $p=4,0455$. На рис. 5 изображены графики решения $y(t)$ и $x(t)$ при значении параметра $p=-3,8655$, с шагом метода Эйлера $h=0,0001$. На рис. 6 изображены графики решения $y(t)$ и $x(t)$ при значении параметра $p=4,0455$, с шагом метода Эйлера $h=0,0001$.

5. Выводы. Применение наилучшей параметризации в ходе метода стрельбы позволяет найти возможные решения задачи, когда система является сингулярно возмущенной и содержит предельные точки, в то время как другие численные методы могут расходиться. Несмотря на то, что применение метода непрерывного продолжения по наилучшему параметру при решении задачи Коши осложняется преобразованием исходной задачи, а также увеличенным временем счета, но если кривая множества решений содержит предельные особые точки, то предложенный подход позволяет эффективно построить решения исследуемой задачи при найденных значениях параметров метода стрельбы. 


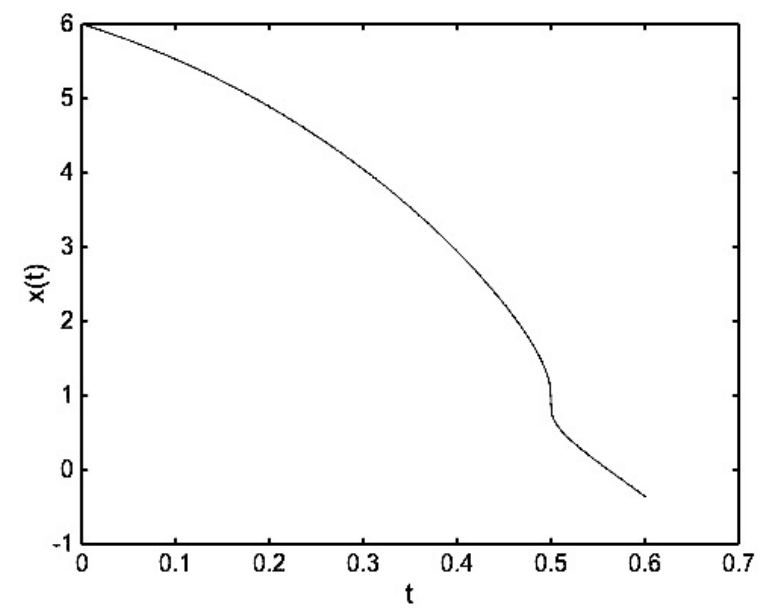

Рис. 3. График решения $x(t)$

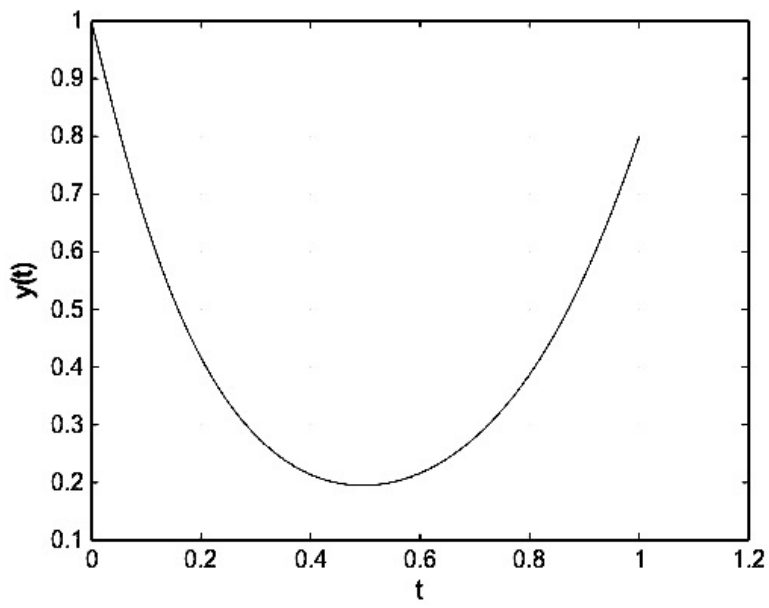

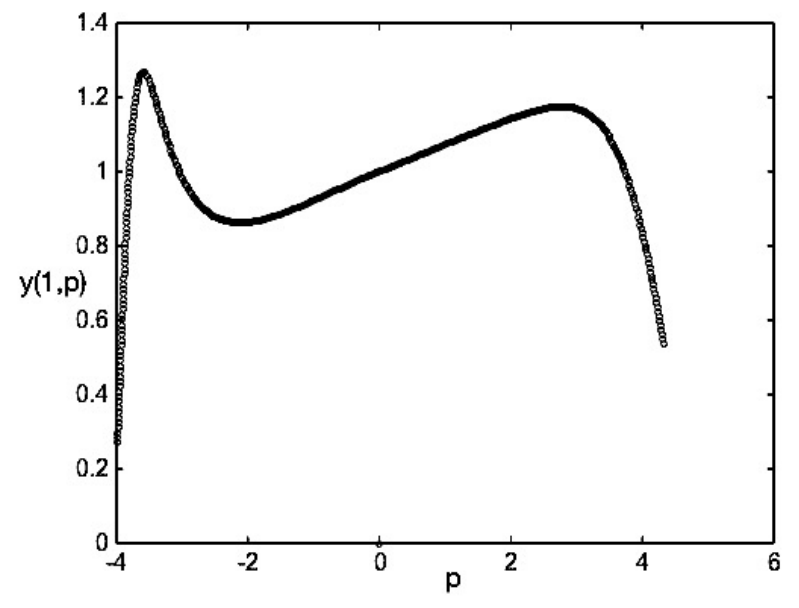

Рис. 4. График решения $y(1, p)$

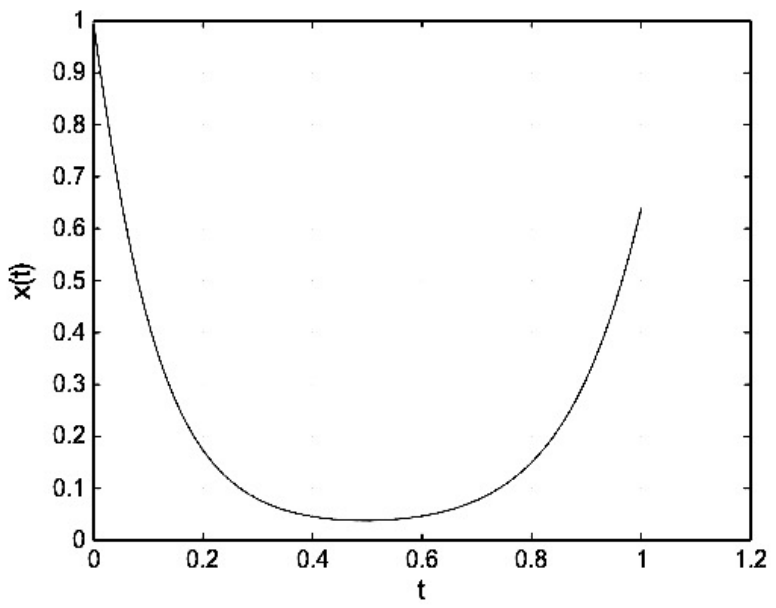

Рис. 5. Графики решения $y(t)$ и $x(t)$ при $p=-3,8655$.
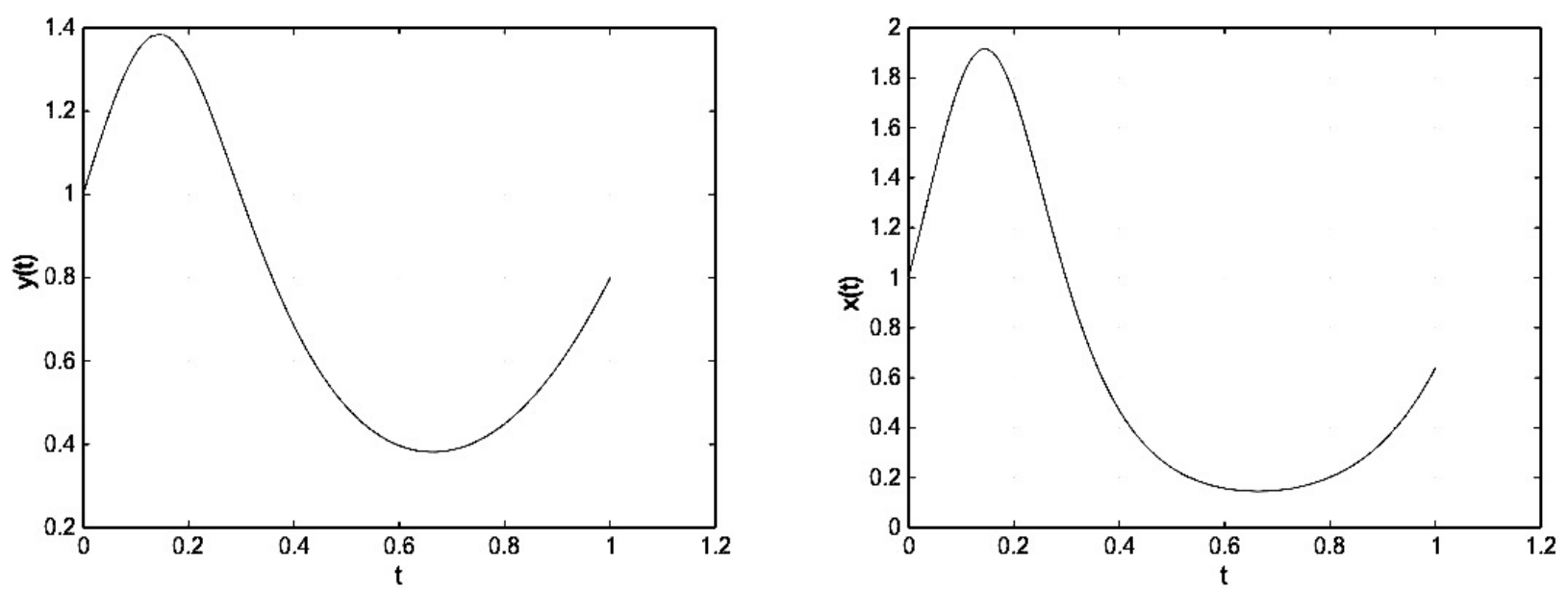

Рис. 6. Графики решения $y(t)$ и $x(t)$ при $p=4,0455$. 


\section{СПИСОК ЛИТЕРАТУРЫ}

1. Бахвалов Н. С., Жидков Н. П., Кобельков Г. М. Численные методы. - М.: Наука, 1987.

2. Будкина Е. М., Кузнецов Е. Б. Численное решение краевых задач для систем интегродифференциально-алгебраических уравнений// Мат. ХІ Междунар. конф. по неравновесным процессам в соплах и струях (NPNJ'2016) (Алушта, 25-31 мая 2016 г.). - М.: Изд-во МАИ, 2016. - С. 417-419.

3. Будкина Е. М., Кузнещов Е. Б. Моделирование технологического процесса производства узлов летательных аппаратов на основе наилучшей параметризации краевой задачи для нелинейных дифференциально-алгебраических уравнений// Вестн. МАИ. - 2016. - 23, № 1. - С. 189-196.

4. Дмитриев C. C., Кузнецов Е. Б. Численное решение систем интегродифференциально-алгебраических уравнений с запаздывающим аргументом// Ж. вычисл. мат. мат. физ. -2008 . -48 , № 3 . C. $430-444$.

5. Каменский Г. А., Скубачевскиий А. Л. Линейные краевые задачи для дифференциально-разностных уравнений. - М.: Изд-во МАИ, 1992.

6. Красников С. Д., Кузнещов Е. Б. Параметризация численного решения краевых задач для нелинейных дифференциальных уравнений// Ж. вычисл. мат. мат. физ. - 2005. - 45, № 12. - С. 2148-2158.

7. Кузнецов Е. Б. Наилучшая параметризация при построении кривых// Ж. вычисл. мат. мат. физ. 2004. - 44, № 9. - C. 1540-1551.

8. Самойленко А. М., Ронто Н. И. Численно-аналитические методы исследования решений краевых задач. - Киев: Наукова Думка, 1986.

9. Шалашилин В. И., Кузнецов Е. Б. Метод продолжения решения по параметру и наилучшая параметризация в прикладной математике и механике. - М.: Едиториал УРСС, 1999.

10. Lahaye M. E. Une metode de resolution d'une categorie d'equations transcendentes// C. R. Hebdomat. Seances Acad. Sci. — 1934. — 198, № 21. — P. 1840-1842.

Афанасьева Мария Николаевна

Московский авиационный институт (национальный исследовательский университет)

E-mail: mary.mai.8@yandex.ru

Кузнецов Евгений Борисович

Московский авиационный институт (национальный исследовательский университет)

E-mail: kuznetsov@mai.ru 